\title{
Recommendations for the medical task force against COVID-19: Zhongshan experience in Wuhan
}

\author{
Zhe Luo ${ }^{1,2 \#}$, Guo-Wei Tu ${ }^{1 \#}$, Qing Yu ${ }^{3}$, Yu-Meng Yao ${ }^{4}$ Yuan Xue ${ }^{5}$, Xiang Chen ${ }^{4}$, Min Fei ${ }^{6}$, Chou-Wen Zhu ${ }^{7}$ \\ ${ }^{1}$ Department of Critical Care Medicine, Zhongshan Hospital, Fudan University, Shanghai, China; ${ }^{2}$ Department of Critical Care Medicine, Xiamen \\ Branch, Zhongshan Hospital, Fudan University, Xiamen, China; ${ }^{3}$ Department of Rehabilitation, Zhongshan Hospital, Fudan University, Shanghai, \\ China; ${ }^{4}$ Department of Infection Control, Zhongshan Hospital, Fudan University, Shanghai, China; ${ }^{5}$ Department of Emergency, Zhongshan \\ Hospital, Fudan University, Shanghai, China; ${ }^{6}$ Department of Anesthesiology, Zhongshan Hospital, Fudan University, Shanghai, China; ${ }^{7}$ Department \\ of Gastroenterology, Zhongshan Hospital, Fudan University, Shanghai, China \\ "These authors contributed equally to this work. \\ Correspondence to: Chou-Wen Zhu. Zhongshan Hospital, Fudan University, Shanghai 200032, China. Email: zhu.chouwen@zs-hospital.sh.cn.
}

Submitted Jul 27, 2020. Accepted for publication Oct 30, 2020.

doi: $10.21037 /$ atm-20-5534

View this article at: http://dx.doi.org/10.21037/atm-20-5534

The COVID-19 outbreak commenced in late December 2019, and rapidly triggered a public health crisis in China, especially in Wuhan. Although the local government sought to handle the crisis, persistently increasing cases "overwhelmed" hospitals and health care workers (1). Therefore, task forces from other regions of China were called on to manage critically ill patients with COVID-19. These teams were organized by the top hospitals, and comprised physicians from different disciplines. Thus, it was important to integrate and rapidly assemble all staff into efficient teams.

Zhongshan Hospital is one of the top five hospitals in mainland China. The hospital dispatched a medical team to Wuhan in February 2020. The team was led by intensivists and consisted of 130 health care workers from twelve disciplines, including nurses, respiratory physicians, emergency physicians, respiratory therapists and thoracic surgeons. The rescue team took over two COVID-19 wards of Hubei General Hospital. Since then, we reviewed our experiences in Wuhan and assembled recommendations for global task forces to combat COVID-19 in their own jurisdictions.

\section{Intensivists as leaders}

As most team members had limited experience in intensive care, a professor of critical care medicine was commissioned as the team leader. On every shift, 3 physicians were on duty in COVID-19 ward with 40 beds, and at least one intensivist or anesthesiologist was allocated to each shift. Intensivists were also assigned to take charge of the most critically ill patients, especially those under mechanical ventilation, continuous renal placement therapy (CRRT) and extracorporeal membrane oxygenation (ECMO) (1). Intensivists and anesthesiologists performed the majority of invasive procedures, such as endotracheal intubation, tracheotomy and central venous catheterizations.

\section{Formulating a standard operating procedure (SOP) for protection}

Our medical team included healthcare-associated infection control physicians who initially established infection prevention and control strategies. The main task of the group was to prevent infection of health care workers, with a "zero infection" goal. To achieve this goal, these strategies covered wards, vehicles and residential areas to form a closed loop. Sometimes, in the case of lacking hardware conditions, infection prevention and control work must be combined with the actual situation to eliminate the potential risk of infection $(2,3)$.

\section{Wireless networks}

In considering the safety of healthcare workers, and limited personal protective equipment (PPE), we restricted healthcare workers in "contamination areas". Importantly, wireless networks helped facilitate the quality of care in 


\begin{tabular}{|c|c|c|c|c|c|c|c|c|c|c|}
\hline \multicolumn{11}{|c|}{ Daily Checklist for COVID-19 Ward } \\
\hline \multirow{2}{*}{\multicolumn{2}{|c|}{ PATIENT }} & \multicolumn{9}{|l|}{$\operatorname{PCR}(+):$} \\
\hline & & \multicolumn{9}{|l|}{ PCR(-): } \\
\hline \multicolumn{6}{|c|}{ Admission: } & \multicolumn{5}{|l|}{ Discharge: } \\
\hline \multicolumn{11}{|c|}{ Moderate: } \\
\hline \multicolumn{11}{|c|}{ Severe: } \\
\hline \multicolumn{11}{|c|}{ Critically: } \\
\hline \multicolumn{6}{|c|}{ TODAY EXAM } & \multicolumn{5}{|c|}{ Today PCR } \\
\hline \multicolumn{6}{|l|}{ X-ray: } & \multicolumn{5}{|c|}{ Nasopharyngeal: } \\
\hline \multicolumn{6}{|l|}{ CT: } & \multicolumn{5}{|c|}{ Sputum: } \\
\hline \multicolumn{6}{|l|}{ ECG: } & \multicolumn{5}{|l|}{ BAL: } \\
\hline \multicolumn{6}{|c|}{ Ultrasound: } & \multicolumn{5}{|l|}{ Feces: } \\
\hline \multicolumn{11}{|l|}{ ABG: } \\
\hline \multicolumn{6}{|c|}{ RESPIRATORY SUPPORT } & \multicolumn{5}{|c|}{ SPECIAL CATHETER } \\
\hline \multicolumn{6}{|c|}{ HFNC: } & \multicolumn{5}{|c|}{ CVC: } \\
\hline \multicolumn{6}{|l|}{ NIV: } & \multicolumn{5}{|c|}{ Artery catheter: } \\
\hline \multicolumn{6}{|l|}{ MV: } & \multicolumn{5}{|c|}{ Urinary catheter: } \\
\hline \multicolumn{6}{|c|}{ ECMO: } & \multicolumn{5}{|c|}{ CRRT: } \\
\hline \multicolumn{11}{|c|}{ Daily Checklist for COVID-19 Patients } \\
\hline Bed & $\mathrm{SpO}_{2}$ & $\begin{array}{l}\text { Support/ } \\
\mathrm{FiO}_{2}\end{array}$ & Antivirus & GCs & $\begin{array}{l}\text { Anticoag } \\
\text { ulation }\end{array}$ & Thymosin & IVIG & $\begin{array}{l}\text { Traditional } \\
\text { Chinese } \\
\text { Medicine }\end{array}$ & Transfusion & Notes \\
\hline 1 & 95 & HFNC (85\%) & Arbidol (D2) & - & D1 & D1 & D3 & D3 & RBC & \\
\hline 2 & 93 & $\operatorname{NIV}(60 \%)$ & Arbidol (D3) & $\begin{array}{c}40 \mathrm{mg} \\
\text { q12h (D3) }\end{array}$ & D2 & - & D3 & D2 & - & \\
\hline .. & .. & .. & .. & .. & .. & .. & .. & .. & .. & \\
\hline
\end{tabular}

Figure 1 Daily checklist for COVID-19 patients. PCR, polymerase chain reaction; CT, computed tomography; ECG, electrocardiograph; ABG, arterial blood gas; BAL, bronchoalveolar lavage; HFNC, high-flow nasal cannula oxygen therapy; NIV, non-invasive ventilation; MV, mechanical ventilation; ECMO, extracorporeal membrane oxygenation; CVC, central venous catheter; CRRT, continuous renal replacement therapy; GCs; glucocorticoids; IVIG, intravenous immunoglobulin.

these settings. We used "WeChat", which is a popular social platform in China, to help staff in "contamination areas" and "clean areas" communicate clinical issues. To maintain the highest quality patient management, five experts were available online at all times for consulting, especially for clinical evaluations and major decisions.

\section{Triaging patients}

Patients admitted to wards were triaged into three groups according to disease severity: moderate, severe and critically ill. All patients received a computed tomography $(\mathrm{CT})$ scan before ICU admission, and were followed up with weekly CT scans. We paid particular attention to patient age, respiratory rate (RR), pulse oxygen saturation $\left(\mathrm{SaO}_{2}\right)$ and lymphocyte counts as these parameters were often associated with deteriorating disease progression. Acute Physiology and Chronic Health Evaluation (APACHE) II, CURB-65 and Sequential Organ Failure Assessment (SOFA) were also used to evaluate patient disease severity. Patients requiring high flow nasal cannula (HFNC) therapy, noninvasive ventilation (NIV) and invasive ventilation, were regarded as severe patients. Another reason for subgrouping patients was that rescue teams could rationally allocate their medical resources (e.g., nurse-to-patient ratio) more appropriately.

\section{Setting and updating checklists}

Checklists were used as core practices. Nurses who directly cared for patients shifted every four hours, and those conducting indirect care shifted every eight hours. Physicians adopted the 12 on 12 off working system. A clear and complete daily checklist was extremely important to our work. The checklist was divided into two parts; the overall situation of the ward, and specific key points of each patient (Figure 1). The daily checklist included antiviral, immunotherapy, oxygen therapy and other supportive 


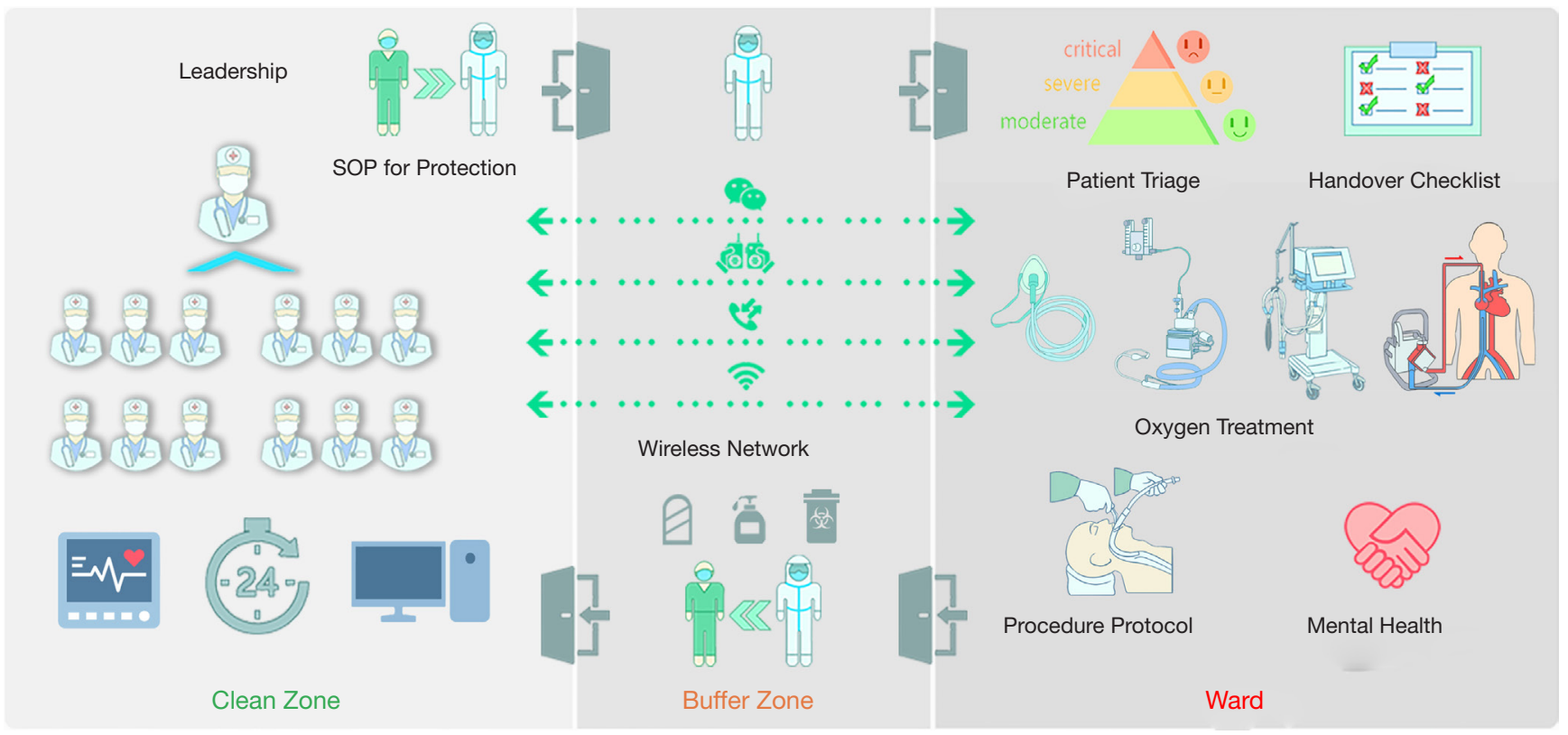

Figure 2 Recommendations for the medical task force against COVID-19.

treatment.

\section{Oxygen therapy}

Oxygen therapy using a nasal cannula or a mask was initiated if arterial $\mathrm{SaO}_{2}$ was $<93 \%$. HFNC or NIV was implemented if patients developed respiratory distress (often $\mathrm{RR}>30$ breaths/min), depending on the preference of the attending physician, however, HFNC was more frequently used due to increased patient tolerance. If the patient developed persistent hypoxemia $\left(\mathrm{PaO}_{2} / \mathrm{FiO}_{2} \leq 150 \mathrm{mmHg}\right)$, and respiratory distress could not be alleviated using HFNC or NIV, invasive mechanical ventilation was indicated. Protective lung ventilation ( $\mathrm{Vt} \leq 6 \mathrm{~mL} / \mathrm{kg}$ predicted body weight) was routinely implemented and positive end expiratory pressure (PEEP) was personally titrated. According to the guidelines and our clinical practice, we recommend prone positioning in patients with severe hypoxemia $(4,5)$.

\section{Protocols for high risk procedures}

Preparing medical respirators, goggles, protective clothing, protective face shields and gloves is essential before procedures. When possible, we used positive pressure medical protective headgear, powered by an air purifier. The number of people in a room should be minimized to those specifically required by patients. If no negative pressure wards are available, rooms should be well ventilated. After the procedure was complete, contaminated equipment and items should be properly disposed of, sealed off and discarded, or thoroughly disinfected. Attention should be paid to strict hand hygiene. Transportation of critically ill patients was also regarded as the high-risk procedure requiring full preparation.

\section{Interventions for mental health problems}

Based on mental health surveys in groups affected by COVID-19, confirmed cases and frontline medical staff were considered high-risk groups in developing mental problems. Those frontline healthcare workers directly treating patients, frequently endured depression, anxiety, insomnia and distress symptoms (6). Daily chronicling of health status and related adverse events in these workers, included mental and physical health evaluations. A psychologist was available $24 \mathrm{~h} / 7$ days a week, either on-site or by telephone counseling.

In summary, the COVID-19 epidemic has placed a huge burden on global health care systems. Eight recommendations for the medical task force against COVID-19 are summarized in Figure 2. We hope our experiences fighting COVID-19 in Wuhan are helpful for task forces in other regions. 


\section{Acknowledgments}

We thank all the colleagues in the task force against COVID-19 epidemic in Wuhan.

Funding: This article was supported by Program of Shanghai Academic/Technology Research Leader (20XD1421000), Natural Science Foundation of Shanghai (20ZR1411100), National Natural Science Foundation of China (82070085) and the Research Funds of Shanghai Municipal Health Commission (2019ZB0105).

\section{Footnote}

Provenance and Peer Review: This article was a free submission to the journal. The article did not undergo external peer review.

Conflicts of Interest: All authors have completed the ICMJE uniform disclosure form (available at http://dx.doi. org/10.21037/atm-20-5534). GWT serves as an unpaid section editor of Annals of Translational Medicine. The other authors have no conflicts of interest to declare.

Ethical Statement: The authors are accountable for all aspects of the work in ensuring that questions related to the accuracy or integrity of any part of the work are appropriately investigated and resolved.

Open Access Statement: This is an Open Access article distributed in accordance with the Creative Commons Attribution-NonCommercial-NoDerivs 4.0 International
License (CC BY-NC-ND 4.0), which permits the noncommercial replication and distribution of the article with the strict proviso that no changes or edits are made and the original work is properly cited (including links to both the formal publication through the relevant DOI and the license). See: https://creativecommons.org/licenses/by-nc-nd/4.0/.

\section{References}

1. Li L, Gong S, Yan J. Covid-19 in China: ten critical issues for intensive care medicine. Crit Care 2020;24:124.

2. Siow WT, Liew MF, Shrestha BR, et al. Managing COVID-19 in resource-limited settings: critical care considerations. Crit Care 2020;24:167.

3. Vergano M, Bertolini G, Giannini A, et al. Clinical ethics recommendations for the allocation of intensive care treatments in exceptional, resource-limited circumstances: the Italian perspective during the COVID-19 epidemic. Crit Care 2020;24:165.

4. Guerin C, Beuret P, Constantin JM, et al. A prospective international observational prevalence study on prone positioning of ARDS patients: the APRONET (ARDS Prone Position Network) study. Intensive Care Med 2018;44:22-37.

5. Gattinoni L, Chiumello D, Rossi S. COVID-19 pneumonia: ARDS or not? Crit Care 2020;24:154.

6. Lakhani A. Which Melbourne Metropolitan Areas Are Vulnerable to COVID-19 Based on Age, Disability, and Access to Health Services? Using Spatial Analysis to Identify Service Gaps and Inform Delivery. J Pain Symptom Manage 2020;60:e41-e44.
Cite this article as: Luo Z, Tu GW, Yu Q, Yao YM, Xue Y, Chen X, Fei M, Zhu CW. Recommendations for the medical task force against COVID-19: Zhongshan experience in Wuhan. Ann Transl Med 2020;8(23):1618. doi: 10.21037/atm20-5534 